\title{
Grid supports design for dual-cooled fuel rods
}

\author{
JaeYong Kim, YoungHo Lee, KyungHo Yoon and \\ HyungKyu Kim \\ Innovative Nuclear Fuel Division, Korea Atomic Energy Research Institute \\ 1045 Daedeok-daero, Youseong-gu, Daejeon, 305-353, Korea \\ kjykjy@kaeri.re.kr
}

\section{INTRODUCTION}

To enhance fuel safety and to achieve a power uprating, a new concept PWR (Pressurized Water Reactor) fuel, which is called a dual cooled fuel rod, has been studied since 2007. Although there are some challenging problems about each mechanical component, fuel rod supporting structures are especially dealt within this paper. In the dual-cooled annular fuel rod, an inner flow passage as well as an outer one is used not only to enhance the fuel safety but to achieve a power uprating through the decreasing fuel center temperature and increasing the heat transfer area between fuel and coolant. As a result, the diameter of dualcooled fuel rods becomes 1.6 times bigger than a conventional solid fuel rod and the gap between the annular fuel and the spacer grid s straps is narrowed. This is a current key issue of fuel rod supports [1]. To accommodate the narrowed gap, spacer grids, such as the cantilever type, hemi-sphere type [2], circular insertion type [3], etc., were suggested. For some of these configurations, patents have been applied.

The grid springs in a spacer grid play the role of holding the fuel rods in an appropriate position and preventing fuel rods from dropping during normal reactor operation. In the case of the dual cooled fuel rod, the total mass is increased. So, the stiffness of a grid spring has to be increased more than that of a conventional grid spring. However, if the stiffnesses of springs are increased too much, fuel rods can bend due to the prohibition of their axial slip. So, it is necessary to design an appropriate stiffness.

In this paper, the minimum spring force to prevent dual-cooled fuel rods from dropping during normal reactor operation is calculated. The spring characteristics of a cantilever type and a hemi-sphere type are predicted. A finite element analysis is carried out by using the commercial code ABAQUS. The analysis results are verified by experiments. Finally, it is checked whether the property of the suggested springs satisfies the minimum required spring force. Based on the obtained results, a kind of spacer grid candidate for dual cooled fuel rods, i.e. a spacer grid with hybrid supports is suggested.

\section{MINIMUM SPRING FORCE}

The mass of a dual cooled fuel rod is calculated conservatively at about $4.899 \mathrm{~kg}$. If $n$ spacer grids are used in a fuel assembly, the force to hold a fuel rod per unit cell will be calculated by using the law of friction.

$$
\begin{gathered}
F=\mu N \\
N \geq \frac{4.899}{\mu n}[k g]=\frac{16.33}{n}[k g]=\frac{160.034}{n}[N]
\end{gathered}
$$


Here is each parameter:

$n$ : the number of spacer grids

$\mu$ : the friction coefficient between supports and fuel rods ( 0.3 is used here)

$N$ : normal force.

The number of spacer grids is designed as 9 11, then the holding force of the unit cell is about $14.55 \sim 17.78 \mathrm{~N}$. If there are 4 supports in a unit cell, the minimum force for one support is $3.64 \sim 4.45 \mathrm{~N}$. And then, the relaxation ratio of the elastic modulus at the operating temperature, $320 \mathrm{i}$, is $70 \%$ of that at the normal temperature. Finally, the required spring force of a support has to be larger than 5.2 6.36 N. Because irradiated supports usually have loosed spring force and become brittle, the radiation effect is of no concern.

\section{GOVERNING EQUATION FOR SUPPORTS}

Fig. 1 shows some parameters which have to be decided for a hemi-sphere support. Each parameter is listed below:

- $\quad D$ : the diameter of a fuel rod

- $d_{s}$ : the diameter of a grid spring

- $H$ : the height of a grid cell

- $h$ : the height of a grid spring

- $\quad Y$ : the distance between the center of a grid spring and the nearest plate

- $\quad X$ : the distance between the centers of a fuel rod and a grid spring

- $\delta$ : the initial interference between a fuel rod and a grid spring

- $t$ : the thickness of a grid spring (equal to the thickness of straps)

The following equation is constituted to determine the parameters. $D, H$ and $t$ are constants. $d_{s}, h$ and $Y$ are to be decided for desired $\delta$. These four parameters are dependent on each other. $\delta$ is decided to obtain suitable stiffness.

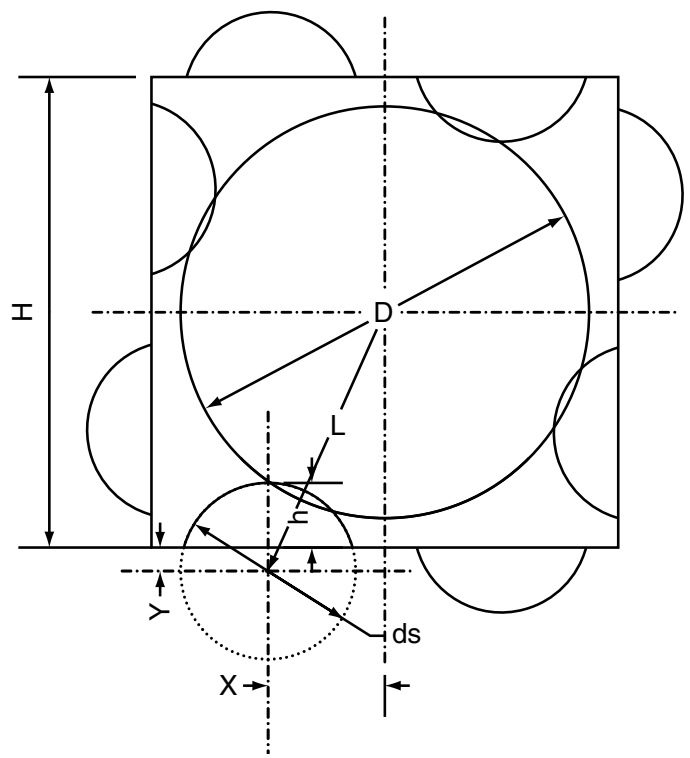

Figure 1 Schematic drawing of hemi-sphere supports. 


$$
\begin{aligned}
& \delta=\frac{D}{2}+\frac{d s}{2}+\frac{t}{2}-L \\
& L=\sqrt{X^{2}+\left(\frac{H}{2}+Y\right)^{2}}
\end{aligned}
$$

By using Eq. (1) and (2), the governing equation for hemi-sphere supports is finally obtained as:

$$
X=\sqrt{\left(\frac{D}{2}+\frac{H}{2}+d s+\frac{t}{2}-\delta-h\right)\left(\frac{D}{2}-\frac{H}{2}+\frac{t}{2}-\delta+h\right)}
$$

By using Eq. (2), hemi-sphere type supports can be designed.

In the case of a cantilever support, Fig. 2 shows some parameters which have to be decided. Each parameter is listed below:

- $\quad D$ : the diameter of a fuel rod

- $\quad r_{2}$ : the radius of a grid spring

- $\quad H$ : the height of a grid cell

- $h$ : the height of a grid spring

- $\theta$ : the degree of the first curve for a cantilever support

- $\quad X$ : the distance between the starting point and final point of a support

- $\delta$ : the initial interference between a fuel rod and a grid spring

- $t$ : the thickness of a grid spring (equal to the thickness of straps)

The following equation is constituted to determine the parameters. $D, H$ and $t$ are constants. $r_{2}, h, \theta$ and $X$ are to be decided for desired $\delta$. These five parameters are dependent on each other. $\delta$ is decided to obtain suitable stiffness.

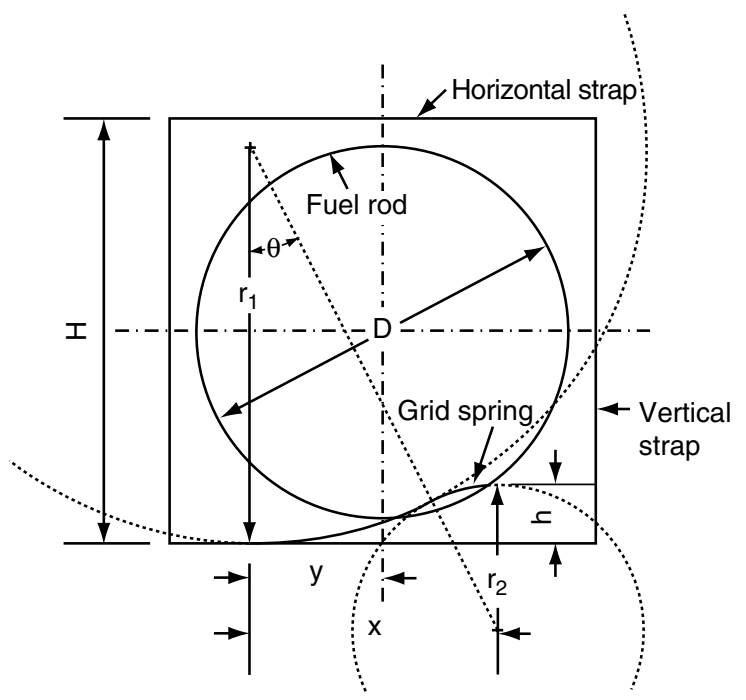

Figure 2 Schematic drawing of cantilever supports. 


$$
\begin{aligned}
& h=\left(\frac{D}{2}+r_{2}\right)(1-\cos \theta) \\
& X=\left(\frac{D}{2}+r_{2}\right) \sin \theta
\end{aligned}
$$

The governing equation for cantilever supports is finally obtained as:

$$
L=\sqrt{\left(\frac{H}{2}+\left(r_{2}-h\right)\right)^{2}+(X-2)^{2}}
$$

\section{SPRING CHARACTERISTIC ANALYSIS AND SPRING CHARACTERISTIC TEST IN GRID CONDITION}

To obtain the spring characteristics in grid condition, a $4 \times 4$ partial model was used. Elasticplastic analysis was performed. The analysis was composed of two steps: shrink fit and loading process [4]. During shrink fit, the circular analytical rigid elements simulated as fuel rods pushed back a grid spring by an initial interference between a fuel rod and a grid spring. This interference is one of the parameters to decide the spring stiffness. During this process, the curved rigid element, simulated as a loading bar, was made as a partial model and apart from a grid spring to prevent contact with other supports. Reference nodes were assigned to rigid elements to control them. Especially, the reference node assigned to the curved rigid element was used to apply a specified deformation of $0.7 \mathrm{~mm}$ downward and to obtain the reaction force. There was a rigid plate to provide the boundary condition. This plate was tied with the lowest plate of a $4 \times 4$ grid model. 6 degree of freedom such as 3 transitions and 3 rotations of its reference node were constrained to prevent rigid body motion. Reaction force and displacement was obtained. Two kinds of strap materials, the SS-304 and the Zry4 , were considered in the analysis. Table 1 summarizes the analytic results of the two types of springs for dual-cooled fuel.

Experimental specimens using decided parameters were made of SS-304 in a 4 by 4 partial model. The spring characteristic test was performed until the maximum load was reached at $30 \mathrm{~N}$ and repeated five times [5]. Table 1 shows the results of the spring characteristic test conducted with the cantilever and hemi-sphere type supports.

In the case of the cantilever type, the ratio of analysis to experimental results was about 0.69 . It is 0.73 in the case of the hemi-sphere type. In the results, it should be noted that the

Table 1 Numerical results

\begin{tabular}{lccrrr} 
Model & \multicolumn{2}{c}{ Cantilever } & \multicolumn{2}{c}{ Hemi-sphere } & Hybrid \\
\hline Material & SS-304 & Zry-4 & SS-304 & Zry-4 & Zry-4 \\
\hline Interference [mm] & 0.32 & 0.32 & 0.2 & 0.2 & 0.31 \\
\hline Loading force [N] & 16.8 & 8.9 & 101.6 & 83.0 & 40.9 \\
\hline Stiffness [N/mm] & 47.8 & 30.3 & 973.8 & 599.2 & 47.9 \\
\hline
\end{tabular}


Table 2 Experimental spring stiffness [N/mm]

\begin{tabular}{lcc} 
Model & Cantilever & Hemi-sphere \\
\hline $1^{\text {st }}$ & 68.6 & 1039.5 \\
\hline $2^{\text {nd }}$ & 66.7 & 1437.6 \\
\hline $3^{\text {rd }}$ & 70.7 & - \\
\hline $4^{\text {th }}$ & 69.7 & - \\
\hline $5^{\text {th }}$ & - & 1504.1 \\
\hline mean & 68.9 & 1327.1 \\
\hline
\end{tabular}

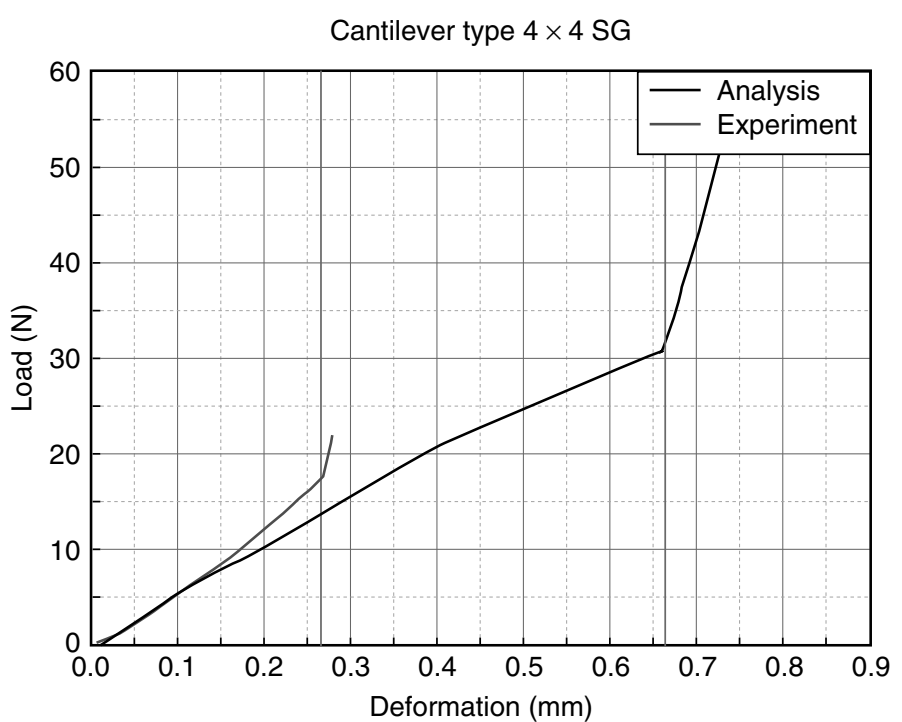

Figure 3 Characteristic curves of the cantilever spring.

loading force is the same as the initial spring force. The characteristic curves of spring obtained from the experiment and analysis are shown as Fig. 3. Both results coincide well until the deformation reaches $0.15 \mathrm{~mm}$. Some discrepancy appears beyond $0.15 \mathrm{~mm}$. The sharply increasing region is attributed to the contact of the loading bar and base plate of supports. If the contact occurs, the loading bar pushes the fuel rod (or circular rigid element) of the adjacent cell. Finally the stiffness difference between the analysis and experiment was around $30 \%$. It is attributed to the plastic property of SS-304, non-uniform welding bead, manufacturing errors and test errors.

\section{SPACER GRID WITH HYBRID SUPPORTS FOR DUAL COOLED FUEL ASSEMBLY}

Experimental results are for SS-304 spacer grids. However, the Zry-4 is usually used as the material of spacer grids. So the characteristics of the spring made of Zry-4 have to be obtained. The calculation results are added in Table 1. Although the relationship between the test and analysis may include nonlinearity, we could at least predict the trend of the Zry-4 spring. The analytic results obtained from the pre-established in-grid spring analysis model 


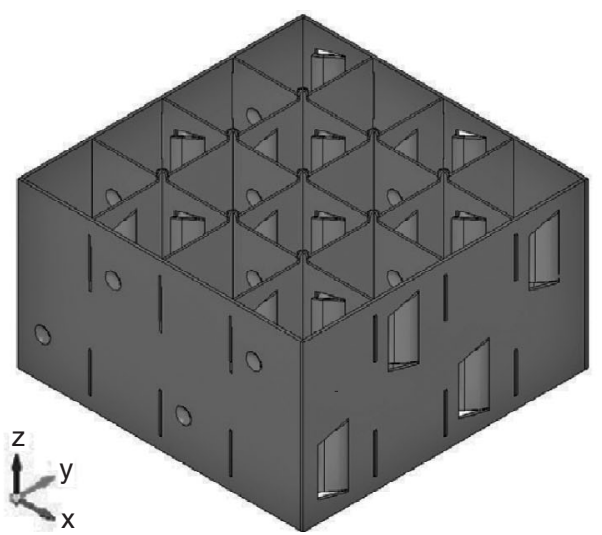

(a) Iso view

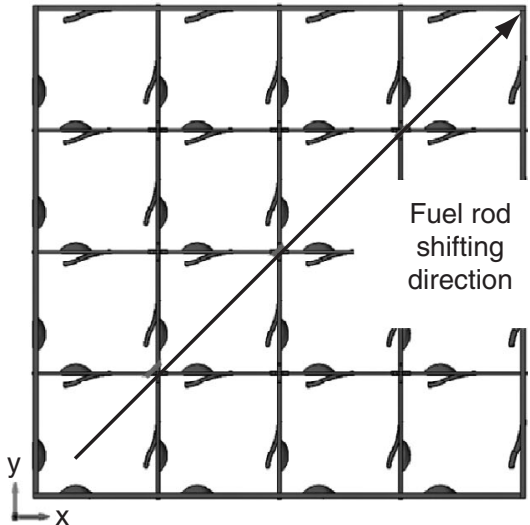

(b) Top view

Figure 4 Partial spacer grid with hybrid supports.

were smaller than the experimental results by about 30\%. Although the suggested spacer grids, especially the cantilever support for dual-cooled fuels could satisfy the minimum required spring force, the difference between the stiffness of the cantilever type and the required minimum spring force is smaller than that of the hemi-sphere type. To achieve greater stiffness of the cantilever type, the $y$ distance is reduced. And because the stiffness of the hemi-sphere type is too big to be a role of a spring, two cantilever type supports for spring and two hemi-sphere type supports for the dimple are used in a unit cell. This is the hybrid supports for dual cooled fuel rods as shown in Fig. 4. The characteristics of hybrid supports become 5 times bigger than the previous cantilever type. Those are summarized in Table 1.

\section{CONCLUSION}

A minimum spring force to prevent the dual-cooled fuel rod from dropping is evaluated. When considering the relaxation ratio of the elastic modulus at the operating temperature, $320 i \mathrm{C}$, the minimum required spring force is about 5.2 6.36 N. The governing equations for cantilever and hemi-sphere type supports are induced for spring parameter decision. The ratios of analysis in the case of the cantilever and the hemi-sphere type to test results are about 0.69 and 0.73 , respectively. The spring stiffness predicted by the finite element analysis was lower than the experimental results by around $30 \%$, but the trend of each parameter s variation was acceptable. Although the predicted stiffness of a cantilever support made of Zry-4 could satisfy the minimum required spring force, it was too low to be a role of a spring until the EOL (End Of Life) of the fuel assembly. To achieve greater stiffness of the cantilever type, a parameter study is performed. And a spacer grid with hybrid supports, two modified cantilever supports for holding the fuel rod and two hemi-sphere supports for positioning the fuel rod is suggested. Finally, the suggested hybrid supports for dual cooled fuel rods made of the Zry-4 satisfy the required spring force and become 5 times bigger.

\section{ACKNOWLEDGEMENTS}

This project has been carried out under the nuclear R\&D program by the Ministry of Education, Science and Technology of Korea. 


\section{REFERENCES}

[1] Hyung-Kyu Kim, Kyung-Ho Yoon, Young-Ho Lee, Jae-Yong Kim and Kang-Hee Lee, Technical Challengers for Dual-Cooling Fuel Structural Components, Proceeding of KNS 2007 spring.

[2] JaeYong Kim, KangHee Lee, KyungHo Yoon and HyungKyu Kim, Parametric Study of CenterMoved Supports of a Spacer Grid, Proceeding of KNS 2008 autumn.

[3] Jae-Yong Kim, Hyung-Kyu Kim, Kyung-Ho Yoon, Young-Ho Lee and Kang-Hee Lee, Design of Insert Type Supports for a Tube Bundle of a Large Diameter, Proceeding of KSME 2008 autumn.

[4] Kim, Jae-Yong, Yoon, Kyung-Ho and Kim, Hyung-Kyu, Realistically Improved Finite Element Analysis of Spring Supports in a Nuclear Fuel Spacer Grid, Proceedings of ICONE16, 2008.

[5] Young-Ho Lee, Jae-Yong Kim, Kang-Hee Lee, Kyung-Ho Yoon, Heung-Seok Kang, Hyung-Kyu Kim, Experimental Characteristic Analysis of $4 \times 4$ Partial Supporting Structure for a DualCooled Fuel, Proceeding of KNS 2009 Autumn. 
